\title{
Appendix Perforation, CTCAE
}

National Cancer Institute

\section{Source}

National Cancer Institute. Appendix Perforation, CT CAE. NCI Thesaurus. Code C57125.

Appendix Perforation that is temporally associated with the use of a medical treatment or procedure, and that may or may not be considered related to the medical treatment or procedure. 